\title{
THE INTERSEASONAL TREND IN PHOTOGRAPHIC DOUBLE STARS OBSERVATIONS
}

\author{
O.V.KIYAEVA \\ Pulkovo Observatory, \\ 196140 St Petersburg, Russia \\ e-mail:kov@gaoran.spb.su
}

Photographic observations obtained in Pulkovo and USNO for four visual double stars (ADS 48, 7251, 11632, 12815) have been investigated.

The Pulkovo data were taken from our catalogue (Kisselev et al., 1988) sent to Strasburg. The USNO data were taken from Josties et al. 1969-78, but only uniform automatic measurements were used.

Analysis of (O-C) relative to the orbital motion shows one year period in both series due to systematic seasonal variations in the conditions of observations. The value of the interseasonal trend (a systematic residual of coordinates) reach up to $0.015^{\prime \prime}$ in $\mathrm{X}$ and $0.030^{\prime \prime}$ in $\mathrm{Y}$ for Pulkovo and $0.007^{\prime \prime}$ in $\mathrm{X}$ and $0.015^{\prime \prime}$ in $\mathrm{Y}$ for USNO. These values are almost of the same range as the accidental errors of observations, but they may be comparable with amplitudes of oscillations caused by a probable invisible satellite and thus they should be taken into account.

Comparisons of Pulkovo and USNO observations have been carried out for each star under the study. The relative motions obtained from both series are in close agreement with each other for all stars. This fact is essential for the orbit determinations.

\section{References}

Kisselev, A.A. et al. (1988) The catalogue of relative positions and motions for 200 visual double stars observed with 26-inch refractor at Pulkovo in 1960-1986. Nauka, Leningrad.

Josties, F.J. et al. (1969-78) Photographic Measures of Double Stars. Publ. Naval Obs.Vol. no. 18, 22, 24,pp. 7-104, 7-88, 7-63. 